\title{
Comparison of parameter fitting on the model of irra- diation effects on bystander cells between Nelder-Mead simplex and particle swarm optimization
}

\author{
Fuaada Mohd Siam ${ }^{1}$, Muhamad Hanis Nasir ${ }^{2}$ \\ ${ }^{1,2}$ Department of Mathematical Sciences, Universiti Teknologi, Johor, Malaysia
}

\author{
Keywords \\ NMS \\ PS \\ Optimization \\ Parameter estimation \\ SSE \\ Received: 8 April 2019 \\ Accepted: 9 May 2019 \\ Published: 28 June 2019
}

\begin{abstract}
Study on the biological effects of irradiation becomes important nowadays. Mathematical modeling is one of the interests among researchers due to its ability to explain the dynamics process of irradiation. Some physical parameters cannot be evaluated from the empirical data. Therefore, the aim of this work to estimate parameters of the model of irradiation effects to bystander cells using optimization approaches. We employ two algorithms: Nelder-Mead Simplex (a local optimizer) and Particle Swarm (a global optimizer). We compare the efficiency of two optimization algorithms in optimizing the parameter values of the model. 50 sets of parameters have been estimated, and all sets can match the model simulation and the experimental data with the least sum-squared error (SSE). The graph of model simulation using a set of the estimated parameter from both optimization algorithms shows a good fit with the experimental data. The overall results indicate that Nelder-Mead Simplex (NMS) is better than Particle Swarm (PS) optimization in the aspect of time computing. At the same time, there is no significant difference in the score of SSE and converging iteration to the least SSE.
\end{abstract}

(C) 2019 The Author(s). Published by TAF Publishing.

\section{INTRODUCTION}

The mathematical modeling framework is often developed by considering the understanding of the biological phenomenon, intuition and assumptions. There must be several parameters introduced into the mathematical model that describes the assumptions of the phenomenon investigated. The determination of the parameters' values becomes challenging when doing the process of model simulation of the biological phenomenon [1, 2]. Even though parameters, such as kinetic rate constants, can be measured from experimental methods [1], but in modeling of the biological system, most of the model parameters cannot be measured directly [3]. The unknown parameters that describe the empirical measurement can be estimated by using few approaches such as optimization technique, statistical methods, and state observers $[2,4,5]$. Parameter fitting plays an important role in the process of monitoring the accuracy of a mathematical model.

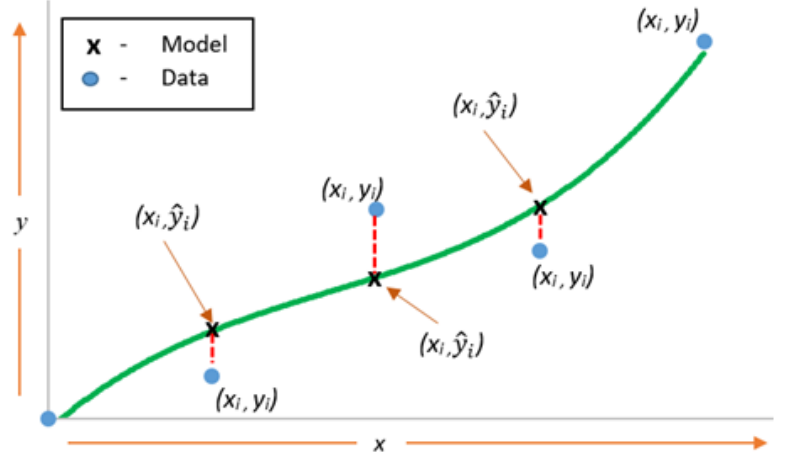

Fig. 1. Difference between the experimental measurements, $y_{i}\left(x_{i}\right)$ and corresponding model simulation data $\widehat{y}_{i}\left(x_{i}\right)$

As shown in Figure 1, parameter fitting identifies the parameter value so that the model's simulation will match with the experimental data. In this paper, the objective function used in the procedure of parameter fitting is called

\footnotetext{
${ }^{*}$ Corresponding author: Fuaada Mohd Siam

†email: fuaada@utm.my
} 
SSE, which is defined as follows:

$$
\mathrm{SSE}=\sum\left[y_{i}\left(x_{i}\right)-\bar{y}_{i}\left(x_{i}\right)\right]^{2}
$$

where $y_{i}$ is the experimental data, $\hat{y}_{i}$ is the model simulation data, and $i=1,2, \ldots, n$ is for $n$ available experimental data set [6]. The experimental data of bystander cells used in this paper is the same as the experimental data used in [7]; see Figure 2.

The SSE minimization Equation 1 is achieved by using the optimization method. The NMS and PS optimizations are employed by considering Equation 1 as the objective function. Here are some studies involving NMS and PS algorithms.

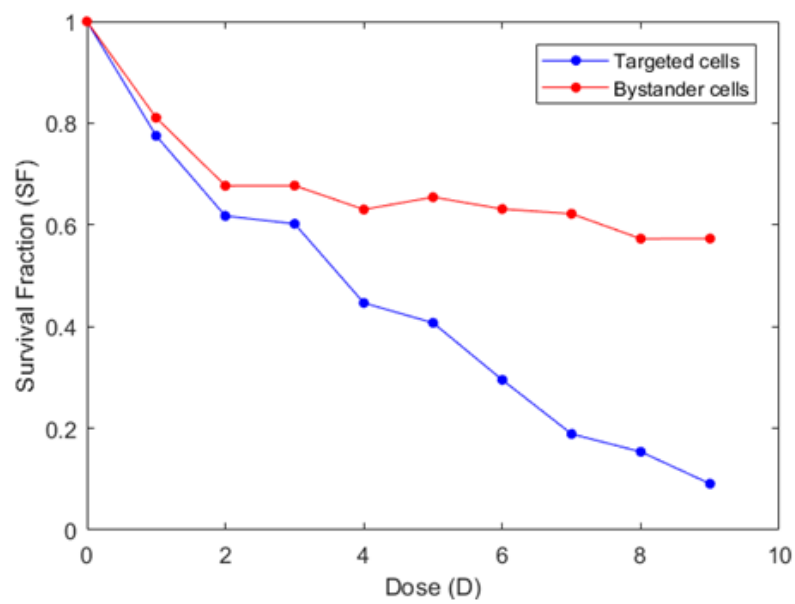

Fig. 2. Experimental data (SF vs. D) of targeted cells and bystander cells [7]

In [8] studied the NMS optimization routine for large-scale problems. They implemented a parallelization towards the sequential algorithm of NMS optimization using a distributed memory implementation. This improvement gave large speedups for computing the NMS optimization for large-scale problems and reducing time-consumption to obtain the result. In [9] employed NMS optimization in order to minimize the stages of operational amplifiers used as filter circuits. In the area of electronics engineering, circuit miniaturization is able to reduce the size of the appliance, power consumption, and increases system reliability. They also compared the NMS optimization with PS optimization and genetic algorithm optimization and showed that PS optimization is the best for their problem.

In [7] used NMS optimization in estimating the value of parameters in the case of high dose effects of irradiation on the targeted cells. Then in [10] showed that NMS optimization is much superior to the Genetic Algorithm in finding the least SEE between the model simulation data and the experimental data. $\operatorname{In}[11]$ studied the distance to the Galactic cen- ter determined by $\mathrm{G}, \mathrm{K}$, and $\mathrm{M}$ stars. The distance from the Sun to the center of Galaxy, $R_{0}$, is a fundamental parameter for the Galactic structure. The kinematical equations of condition contain parameter $R_{0}$, which is in the denominator, making the equation nonlinear. Since the NMS algorithm is a good method dealing with a nonlinear problem, the NMS algorithm has determined the parameters in the nonlinear kinematical equations of condition with the least square error.

In [12] used the PS algorithm technique to identify the unknown parameters of a single diode model of the solar photovoltaic module. Solar energy is directly converted to electricity through a static medium called photo-voltaic. In order to predict the performance of a photo-voltaic system before being installed, a reliable and accurate model with correct parameter value is essential. The result showed that the PS optimization algorithm is able to tackle the problem. In [13] used the PS algorithm to estimate all parameters of an anaerobic glucose digestion model. The results showed that the values of most estimated parameters were close to the reported data. By applying the estimated parameters, the glucose anaerobic glucose digestion model matched the experimental data.

In [14] presented a novel approach to the problem of model checking cyber-physical systems. The model was transformed into an optimization problem by designing an objective function that measures how close a state is to the violation of a property. The minimization of the objective function was achieved by PS optimization. The PS optimization of the model checker quickly found a bug in the controller that could cause the rover to collide with an obstacle. Another research done by [15] used PS optimization and the Genetic Algorithm to optimize the parameters of a three degree-of-freedom model representing the response of the human body to vertical vibration. The optimization result indicated that both optimizations gave close fit to experimental data. They also found that PS optimization is much faster and provides lower mean error than the Genetic Algorithm.

Recently, [7] proposed a mathematical modeling on the irradiation effects on non-targeted cells. The interested reader can refer to $[16,17,18]$ for the information on the phenomenon of irradiation bystander effects towards cells. In [7], the procedure of parameter fitting on the model had been done by using PS optimization. Thus, the intention of this paper is to employ another optimization algorithm that is NMS optimization to be compared with the results from PS optimization. Both optimization algorithms will be compared in terms of SSE value, converging iteration to the 
lowest SSE and the computational time. The significance of the findings can be useful in determining which optimization algorithm is the best for estimating the parameters of the model proposed.

\section{METHODOLOGY}

\section{A. Mathematical Model of Irradiation Effects on By- stander Cells}

The model proposed by [7] contains 8 parameters which are $\vartheta, D_{C}, a_{1}, a_{2}, p, V_{\max }, K_{M}$, and $\tau$. The parameter $\vartheta$ is the Double-Strand Breaks (DSBs) induction coefficient in bystander cells, $D_{C}$ is the characteristic sensitivity of the targeted cells, $a_{l}$ is the death rate due to mis-repair DSBs, $\alpha_{2}$ is the death rate due to two DSBs located close enough to form lethal chromosomal aberrations, $p$ is probability of repair $1 \mathrm{DSBs}, V_{\max }$ is the maximum repair rate, $K_{M}$ is the Michaelis-Mentan constant where $V_{\max }$ is halved and $\tau$ is the repair delay duration. The boundaries for all parameters are obtained from [7] as listed in Table 1. In the procedure of parameter estimation, the parameter will be estimated within these boundaries.

TABLE 1

LIST OF PARAMETER BOUNDARIES [?]

\begin{tabular}{lll}
\hline \hline Parameter & Lower Boundary & Upper Boundary \\
\hline$\vartheta$ & $1.8 C^{-1}$ & $9.7 C^{-1}$ \\
$D_{C}$ & $0.003 \mathrm{~Gy}$ & $3 \mathrm{~Gy}$ \\
$\alpha_{1}$ & $0.0277 h^{-1}$ & $20.79 h^{-1}$ \\
$\alpha_{2}$ & $0 h^{-1}$ & $0.005 h^{-1}$ \\
$p$ & 0 & 1 \\
$V_{\max }$ & $0.1 h^{-1}$ & $3 h^{-1}$ \\
$K_{M}$ & 0 & 5 \\
$\tau$ & $0.05 \mathrm{~h}$ & $6 \mathrm{~h}$ \\
\hline \hline
\end{tabular}

\section{B. Optimization Algorithms}

Optimization is a mathematical discipline that is concerned with finding the maximum or minimum of a function, possibly subject to constraints [19]. The optimization algorithm can be either a local optimizer or a global optimizer, depending on the design and construction of the algorithm [20]. Local optimizer means that the algorithm attempts to find a local minimum, and there is no guarantee to get the global minimum for the problem. A local minimum of a function is a point where the function value is smaller than or equal to the value at nearby points, but possibly greater than at a distant point. In contrast, a global minimum is a point where the function value is smaller than or equal to the value at all other feasible points. However, there are some cases (convex problems like linear programs) where the local minimum found will, in fact, be the global minimum. There can be many local minima which are not global minima [20]; see Figure 3.

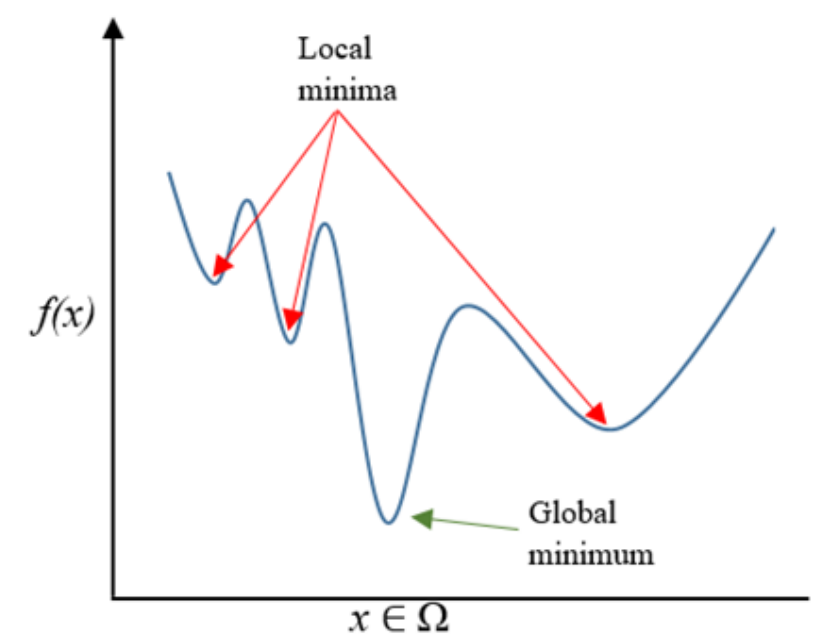

Fig. 3. Local and global minima

Mathematically, given a feasible region $\Omega$ for local optimization, suppose a local minimum $f^{*}=f\left(x^{*}\right)$ with a local minimizer $x^{*} \epsilon \Omega$, the value of $f^{*}$ is the smallest in some feasible neighbourhood. If $f=f(x)$ is also a local minimum with a local minimizer $\times \Omega$, there exists an $\epsilon>0$ such that $f \leq f^{*}$ with $\left|x^{*}-x\right| \leq \epsilon$. For global optimization, suppose a global minimum $f\left(x^{*}\right)$ with a global minimizer $x^{*} \Omega$, the value of $f\left(x^{*}\right)$ is the smallest over all feasible points. That is $f\left(x^{*}\right) \leq f(x)$ for all $\times$ in $\Omega$ [19].

1) The NMS optimization: The NMS is a local optimization that was developed by [21], and it has been widely used in solving problems with irregular objective functions. NMS optimization is able to counter indeterminacies, kinks, discontinuities and local solutions in functions being evaluated [8]. NMS optimization minimizes a function of $\mathrm{n}$ parameters $\left(f(x), x=\left[x_{1}, x_{2}, \ldots, x_{n}\right]\right)$ by comparing function evaluations at the $n+1$ vertices of a general simplex $\left[x^{(1)}, x^{(2)}, \ldots, x^{(n+1)}\right]$, and updating the worst vertex by moving it around a centroid. The simplex may be thought of as a polygon with $n+1$ vertices. If $n=2$, the simplex is a triangle. If $n=3$, the simplex is a tetrahedron [22]. In this work, a built-in MATLAB routine called "fminsearchbnd" is used due to its function as the NMS optimization. The basic geometry in the NMS optimization algorithm is reflection, expansion, contraction and shrinking.

2) PS optimization: The PS is a global optimization that was developed by Kennedy and Eberhart [23], and it has subsequently developed in thousands of scientific papers [24]. PS optimization gained significant popularity due to its simple structure and high performance [25]. PS is swarm 
intelligence inspired by the group behaviour of animals, for example, bird flocks or fish schools. It is a population-based algorithm, that is, it represents the state of the algorithm by a population, which is iteratively modified until termination criteria are satisfied. A reader can refer to [7] for the details on the algorithm of PS optimization. For solving practical problems, the number of particles in a swarm population is usually chosen between 10 and 50 . Using many particles required will cause too many function evaluations per iteration [26]. In this work, a built-in MATLAB function called "particleswarm" is used due to its function as the particle swarm optimization.

\section{RESULTS AND DISCUSSION}

\section{A. Parameter Estimation using NMS and PS Optimiza- tion}

We estimated 50 sets of parameter values (sample set, $n=$ 50) using the NMS optimization. Meanwhile, the results of parameter fitting using the PS optimization are taken from [7] for the purpose of comparison. The statistical analysis on the results of 50 sets of estimated parameters involved the mean, the standard deviation, the confidence interval and the correlation. Table 2 provides the average value of the result of 50 sets of parameter values obtained by both optimization algorithms.

TABLE 2

THE SAMPLE MEAN VALUE $(\bar{X})$ OF EACH ESTIMATED PARAMETER, SSE, AND CORRELATION $(R)$

\begin{tabular}{lll}
\hline \hline Parameter Optimization & Result of NMS & Result of PS by [7] \\
\hline$\vartheta\left(C^{-1}\right)$ & 3.5746 & 3.8001 \\
$D_{C}(G y)$ & 1.6483 & 1.6694 \\
$\alpha_{1}\left(h^{-1}\right)$ & 8.4933 & 10.0548 \\
$\alpha_{2}\left(h^{-1}\right)$ & 0.0024 & 0.0014 \\
$p$ & 0.8358 & 0.8614 \\
$V_{\text {max }}\left(h^{-1}\right)$ & 1.8832 & 1.9514 \\
$K_{M}$ & 3.1561 & 2.5126 \\
$\tau h$ & 1.3067 & 2.0053 \\
SSE & 0.0067 & 0.0053 \\
Correlation $(r)$ & 0.9773 & 0.9825 \\
\hline \hline
\end{tabular}

The average value of SSE tends to be zero. The NMS and PS optimization successfully optimized the model's parameter values with a reasonably small value for the differences between the model's simulation data and the experimental data. The details on the correlation value $(r)$ will be discussed in Section 3.1.4.
1) Sample standard deviation: It is also important to investigate the variations of all parameters based on 50 sets of estimated parameters. The sample standard deviation, $\mathrm{s}$, is a measure of how the data is clustered about the mean [27]. The sample standard deviation is calculated for each parameter for both optimizations, as shown in Table 3.

TABLE 3

\begin{tabular}{lll}
\multicolumn{3}{l}{ ESTIMATED SAMPLE STANDARD DEVIATION $(S)$} \\
\hline \hline Parameter & Optimization Algorithm \\
\cline { 2 - 3 } & NMS & PS \\
\hline$\vartheta$ & 1.8534 & 1.3098 \\
$D_{C}$ & 0.3914 & 0.2085 \\
$\alpha_{1}$ & 6.9547 & 5.3234 \\
$\alpha_{2}$ & 0.0017 & 0.0012 \\
$p$ & 0.0884 & 0.0770 \\
$V_{\max }$ & 0.8993 & 0.8415 \\
$K_{M}$ & 1.3634 & 1.1546 \\
$\tau$ & 1.3121 & 1.5811 \\
\hline \hline
\end{tabular}

The sample standard deviation of parameter ${ }_{1}$ is the largest value compared to the other parameters. This indicates that the sample of the estimated value for parameter ${ }_{1}$ is more spread out than the other seven parameters.

2) Confidence interval of each parameter: The confidence interval provides a method to measure the margin of error 
TABLE 4

for the population mean or population proportion [28]. It is an interval of numbers containing the most plausible values of the population parameters, i.e., the mean, $\mu$ for the entire population is likely to fall in the range. Each parameter stated in the mathematical model (see Table 1) is defined as an independent population parameter. The confidence interval for all eight parameters is calculated. The sample of each population parameter is $n=50$, which corresponds to 50 runs of NMS and PS optimizations for experimental data of the survival fraction of bystander cells.

According to [27], if a random sample is taken from a population that has an unknown probability distribution, the sampling distribution of the sample mean can be approximately normal with mean $\mu$ and variance $\sigma^{2}$, given that if the sample size, $n$, is large. It is referred to as the central limit theorem. If $n \geq 30$, the normal approximation will be satisfactory regardless of the shape of population. In current case, $n=50$ is sufficiently large; hence, the confidence interval takes the form:

$$
\text { Sample mean }+/-[\text { Critical value } \times \text { Standard error }]
$$

Since $n$ is large, the unknown population standard deviation, $\sigma$, can be replaced by sample standard deviation, $s$ (see Table 3).

In this study, $95 \%$ of confidence interval is computed in order to test the reliability of the estimated parameters. Thus, $\alpha=1-0.95=0.05$ and from statistical table of normal distribution (see [27]), the appropriate value for $z_{0.975}$ for area to the left is 1.96. Therefore, the confidence interval can be written as:

$$
\bar{x} \pm 1.96 \frac{s}{\sqrt{n}}
$$

By using Equation 3, the results of the confidence interval for all parameters are presented in Table 4.

From this calculation, it can be interpreted that the true value of the average of each parameter is within this interval with a 95\% confidence level. The parameters of the model are ideally estimated by NMS and PS optimization, with a $95 \%$ confidence level. Specifically, if an infinite number of random samples are collected and (1- $\alpha) 100 \%$ confidence interval for $\mu$ is computed for each sample, $(1-\alpha) 100 \%$ of these intervals will contain the true value of $\mu$ [27]. The estimated parameters will be applied to obtain a simulation data of the model in order to compare the experimental data.
95\% CONFIDENCE INTERVAL OF PARAMETER

\begin{tabular}{lll}
\hline \hline Parameter & Optimization Algorithm & \\
\cline { 2 - 3 } & NMS & PS by [7] \\
\hline$\vartheta$ & $(3.0609,4.0883)$ & $(3.4370,4.1631)$ \\
$D_{C}$ & $(1.5399,1.7568)$ & $(1.6116,1.7272)$ \\
$\alpha_{1}$ & $(5.5656,10.4211)$ & $(8.5792,11.5303)$ \\
$\alpha_{2}$ & $(0.0019,0.0029)$ & $(0.0011,0.0018)$ \\
$p$ & $(0.8113,0.8603)$ & $(0.8401,0.8828)$ \\
$V_{\max }$ & $(1.6339,2.1324)$ & $(1.7181,2.1846)$ \\
$K_{M}$ & $(2.7782,3.5340)$ & $(2.1926,2.8326)$ \\
$\tau$ & $(0.9430,1.6704)$ & $(1.5671,2.4436)$ \\
\hline \hline
\end{tabular}

3) Model simulations using the estimated parameters: As an illustration, figures of simulation between the model simulation data and experimental data of bystander cells are shown in this section.

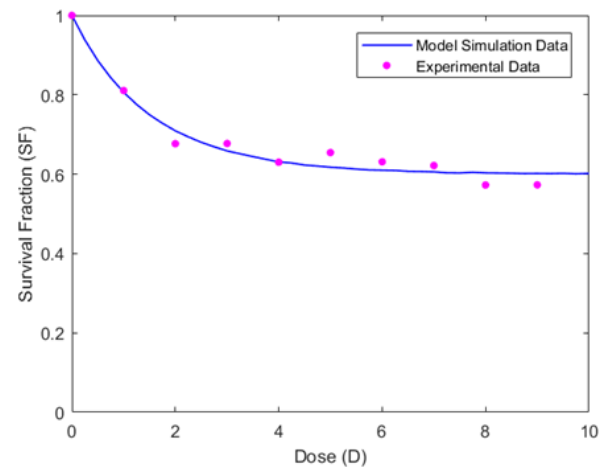

Fig. 4. Data fitting of the experimental data using a set of estimated parameters from NMS optimization. The estimated parameters are: $\vartheta=3.2791$ $C^{-1}, D_{C}=1.6592 \mathrm{~Gy}, \alpha_{1}=0.2168 h^{-1}$, $\alpha_{2}=0.0049 h^{-1}, p=0.8708, V_{\max }$ $=2.4481 h^{-1}, K_{M}=3.6464$ and $\tau$ $=0.0740 h$. The SSE value is 0.0053 and $r=0.9825$

All the results of model simulation (in Figure 4and Figure 5) using the estimated parameters show a good fit between the model simulation and the experimental data of bystander cells. A good fit, by means of the SSE value, is close to 0 and $r$ value near to 1 . Next, the correlation between the model's simulation data and the experimental data will be discussed.

4) Pearson's correlation coefficient: Correlation coefficient $(r)$ is statistics that quantify the relationship between two variables in unit-free terms [29]. The value of $r$ is between $-1 \leq \mathrm{r} \leq+1$. The closer $\mathrm{r}$ to +1 shows stronger positive correlation while the closer $r$ to -1 shows stronger negative correlation.

From Table 2, the mean value of the correlation, $r$, is close to 1 which corresponds to an excellent linear relationship between experimental data and model simulation data. 


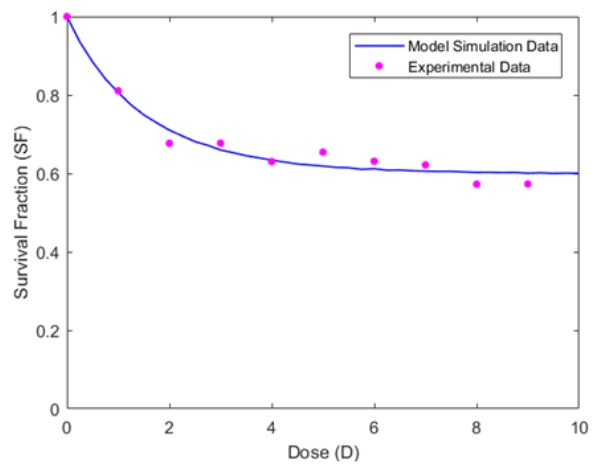

Fig. 5. Data fitting of the experimental data using a one of estimated parameters from PS optimization. The estimated parameters are: $\vartheta=2.2072 C^{-1}, D_{C}$ $=1.7959$ Gy, $\alpha_{1}=7.2906 h^{-1}, \alpha_{2}=$ $0.0020 h^{-1}, p=0.7740, V_{\max }=2.1472$ $h^{-1}, K_{M}=1.9953$ and $\tau=0.0510 h$. The SSE value is 0.0051 and $r=0.9830$

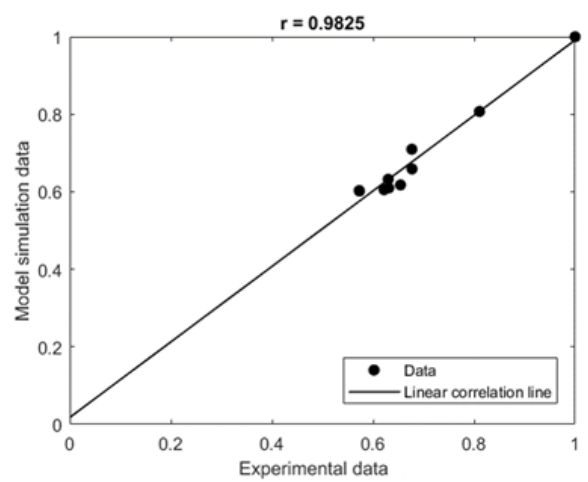

Fig. 6. Linear correlation plot of model simulation data using the estimated parameters from NMS optimization

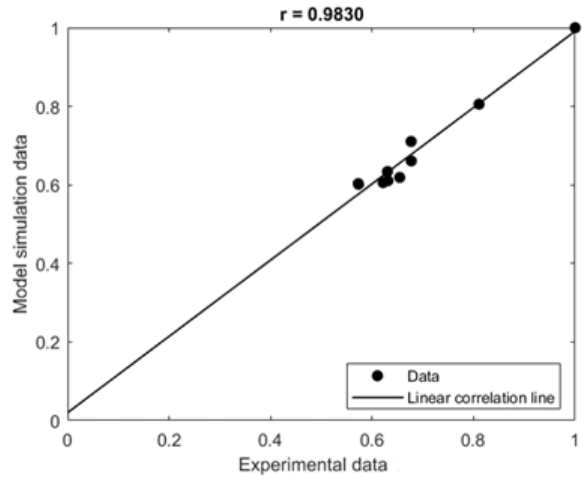

Fig. 7. Linear correlation plot of model simulation data using the estimated parameters from PS optimization

By using the estimated parameters listed in Figures 4 and 5, the corresponding linear correlation plots of model simulation data versus experimental data are presented in Figures 6 and 7, respectively.

\section{B. Comparison between NMS and PS Optimization}

There are three items discussed regarding the efficiency of both optimization algorithms. First there is theability of 
both algorithms to optimize the least SSE and the highest value of $r$ between the model's simulation and the experimental data. Second, the number of iterations is needed to converge to the least SSE. Third, there is average time computing for parameter fitting procedure. Note that the stopping criteria are set to 150 iterations for both optimization algorithms.

In Table 2, the average value of SSE is 0.0067 and 0.0053 for NMS and PS algorithm, respectively. The same goes to the average of correlation value, $r$. The value of $r$ obtained by the PS algorithm is slightly higher than the $r$ value obtained by the NMS algorithm. However, there is not much difference between SSE and $r$ value obtained by both optimization algorithms. For the first item, both optimization algorithms are excellent optimizers to the model developed by [7].

Next, the second item is the number of iterations needed to converge to the least SSE. In order to perform a fair comparison, the same parameter value of initial guess is used for both optimization algorithms. The chosen set of initial guess is parameter set no. 15 in Supplementary File A. The result is presented in Figure 8. It can be seen that PS optimization is much faster in converging to the least SSE value compared to NMS optimization. However, when the iteration is reaching 70 and forward, the SSE value is very close to zero and there is not much difference of SSE score for both optimization algorithms.

For the third item, the average time of parameter fitting procedure over 150 iterations is 3.5647 minutes for NMS optimization, while 22.4017 minutes for PS optimization. Note that both optimization algorithms are run in the same computer specification for the purpose of a fair comparison. The time computing by PS optimization is longer than NMS optimization. The PS optimization requires many function evaluations in each iteration, depending on the number of particle. Every particle in PS algorithm changed position after one iteration, while in NMS algorithm, there is only one point changed after one iteration (except shrinking).

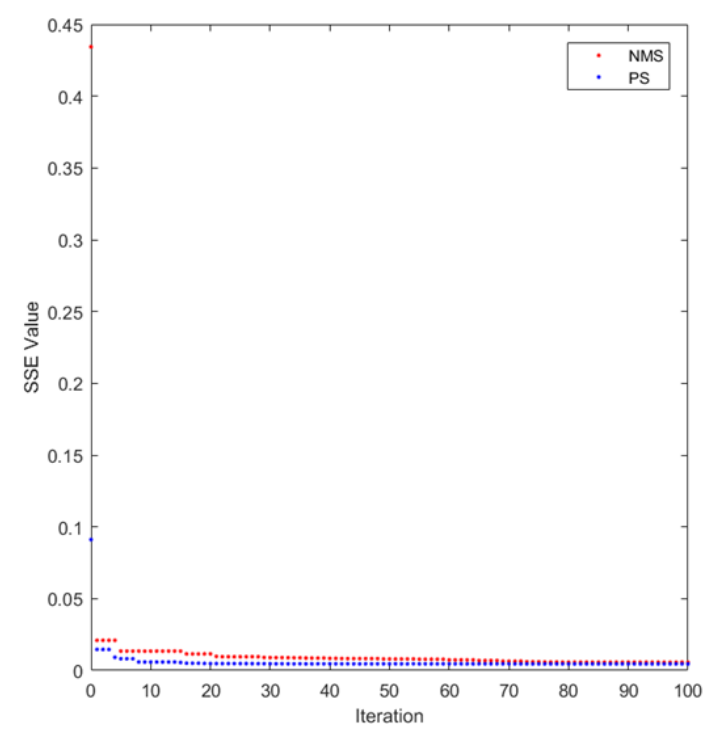

Fig. 8. Linear correlation plot of model simulation data using the estimated parameters from PS optimization

Between the three items discussed regarding the efficiency of both optimization algorithms, it can be seen that there is a huge difference in terms of time computing. The time computing represents the cost that is needed in order to obtain solution for particular problem. Shorter time computing is needed in order to save time and electricity usage. Since there is not much difference for SSE value, $r$ value and converging iteration to the least SSE, it can be concluded that NMS optimization is better than PS optimization because NMS optimization only required shorter time computing.

\section{CONCLUSION}

In this paper, 50 sets of estimated parameters are computed in the parameter fitting procedure. Every set is able to fit the model's simulation data and the experimental data with a minimum value of SSE. Not only that, the correlation coefficient between the experimental data and simulation data is close to 1 for every estimated parameter set, indicating an excellent fit between both simulation and experimental data. The $95 \%$ confidence interval for each parameter also shows that there is a $95 \%$ confidence level that the inter- 
val calculated will have the true average of each parameter. For the comparison of both optimization algorithms, getting results in a shorter time is essential and then put together NMS optimization as the better optimization for the model. For further development, it can be suggested that both algorithms run in a parallel programming interface with high computer specifications for faster function evaluation [30].

\section{ACKNOWLEDGMENT}

This work was financially supported by the Universiti Teknologi Malaysia under the Fundamental Research Grant Scheme (FRGS: R.J130000.7826.4F889) and Ministry of Education Malaysia (MOE).

\section{REFERENCES}

[1] G. Lillacci and M. Khammash, "Parameter estimation and model selection in computational biology," PLoS Computational Biology, vol. 6, no. 3, pp. 45-50, 2010. doi: https://doi.org/10.1371/journal.pcbi.1000696

[2] G. Lillacci and M. Khammash, "A distribution-matching method for parameter estimation and model selection in computational biology," International Journal of Robust and Nonlinear Control, vol. 22, no. 10, pp. 1065-1081, 2012. doi: https://doi.org/10.1002/rnc.2794

[3] B. P. Ingalls, Mathematical Modeling in Systems Biology: An Introduction. Cambridge, MA: MIT Press, 2013.

[4] T. M. Alhamzah, "Design and simulation of optimized QPSK transmitter using HDL code in MATALB," Journal of Advances in Technology and Engineering Studies, vol. 4, no. 5, pp. 207-213, 2018. doi: https://doi.org/10.20474/jater-4.5.4

[5] J. Q. Luo, C. Wei, J. X. Xu, and X. Q. Liang, "Ant colony optimization solutions for path planning of logistic," International Journal of Technology and Engineering Studies, vol. 4, no. 2, pp. 95-101, 2018. doi: https://doi.org/10.20469/ijtes.4. 10003-3

[6] D. Goulet, "Modeling, simulating, and parameter fitting of biochemical kinetic experiments," SIAM Review, vol. 58, no. 2, pp. 331-353, 2016. doi: https://doi.org/10.1137/151004707

[7] F. M. Siam and M. H. Nasir, "Mechanistic model of radiation-induced bystander effects to cells using structured population approach," Matematika, vol. 34, no. 3, pp.149-165, 2018. doi: https://doi.org/10.11113/matematika.v34.n3.1147

[8] K. Klein and J. Neira, "Nelder-mead simplex optimization routine for large-scale problems: A distributed memory implementation," Computational Economics, vol. 43, no. 4, pp. 447-461, 2014. doi: https://doi.org/10.1007/ s10614-013-9377-8

[9] O. J. USHIE, M. ABBOD, E. C. ASHIGWUIKE, and S. LAWAN, “'Constrained nonlinear optimization of unity gain operational amplifier filters using pso, ga and nelder-mead," International Journal of Intelligent Control and Systems, vol. 20, pp. 26-34, 2015. doi: https://doi.org/10.1016/s0020-0190(02)00447-7

[10] F. M. Siam, M. H. A. Kamal, and F. Johar, "Parameter estimation for a mechanistic model of high dose irradiation damage using nelder-mead simplex method and genetic algorithm," Jurnal Teknologi, vol. 78, no. 12-2, pp. 87-92, 2016. doi: https://doi.org/10.11113/jt.v78.10146

[11] R. L. Branham, "The distance to the galactic center determined by g, k, and m stars," Astrophysics and Space Science, vol. 362, no. 2, pp. 29-34, 2017. doi: https://doi.org/10.1007/s10509-017-3015-1

[12] S. Bana and R. Saini, "Identification of unknown parameters of a single diode photovoltaic model using particle swarm optimization with binary constraints," Renewable Energy, vol. 101, no. 6, pp. 1299-1310, 2017. doi: https://doi.org/ 10.1016/j.renene.2016.10.010

[13] J. Yang, L. Lu, W. Ouyang, Y. Gou, Y. Chen, H. Ma, J. Guo, and F. Fang, "Estimation of kinetic parameters of an anaerobic digestion model using particle swarm optimization," Biochemical Engineering Journal, vol. 120, pp. 25-32, 2017. doi: https://doi.org/10.1016/j.bej.2016.12.022

[14] D. Phan, S. A. Smolka, R. Grosu, U. Mehmood, S. D. Stoller, and J. Yang, "Model checking cyber-physical systems using particle swarm optimization," IEEE Engineering Journal, vol. 4, no. 7, pp. 45-60, 2017.

[15] N. Nawayseh, A. Jarndal, and S. Hamdan, "Optimizing the parameters of a biodynamic responses to vibration model using particle swarm and genetic algorithms," in 7th International Conference on Modeling, Simulation, and Applied Optimization, New York, NY, 2017.

[16] M. H. Nasir and F. M. Siam, "Mini-review: recent updates on the mathematical modelling of radiation-induced bystander effects," Malaysian Journal of Fundamental and Applied Sciences, vol. 13, no. 2, pp. 103-108, 2017. doi: https://doi.org/ $10.11113 /$ mjfas.v13n2.563 
[17] R. Wang, T. Zhou, W. Liu, and L. Zuo, "Molecular mechanism of bystander effects and related abscopal/cohort effects in cancer therapy," Oncotarget, vol. 9, no. 26, pp. 186-200, 2018. doi: https://doi.org/10.18632/oncotarget.24746

[18] M. H. Nasir and F. M. Siam, "Simulation and sensitivity analysis on the parameter of non-targeted irradiation effects model," Jurnal Teknologi, vol. 81, no. 1, pp. 45-50, 2019. doi: https://doi.org/10.11113/jt.v81.12448

[19] A. Govan, "Introduction to optimization," in Proceedings of North Carolina State University Undergraduate Workshop, North Carolina, NC, 2006.

[20] J. D. Pintér, Global Optimization in Action: Continuous and Lipschitz Optimization: Algorithms, Implementations and Applications. New York, NY: Springer Science \& Business Media, 2013.

[21] J. A. Nelder and R. Mead, "A simplex method for function minimization," The Computer Journal, vol. 7, no. 4, pp. 308-313, 1965. doi: https://doi.org/10.1093/comjnl/7.4.308

[22] W. H. Press, S. A. Teukolsky, W. T. Vetterling, and B. P. Flannery, Numerical Recipes: The Art of Scientific Computing. Cambridge, UK: Cambridge university press, 2007.

[23] R. Eberhart and J. Kennedy, "A new optimizer using particle swarm theory," in Proceedings of the Sixth International Symposium on Micro Machine and Human Science, New Dehli, India, 1995.

[24] M. R. Bonyadi and Z. Michalewicz, Particle Swarm Optimization for Single Objective Continuous Space Problems: A Review. Cambridge, MA: MIT Press, 2017.

[25] S. Ebbesen, P. Kiwitz, and L. Guzzella, "A generic particle swarm optimization matlab function," in American Control Conference, New Jersy, NJ, 2012.

[26] I. C. Trelea, "The particle swarm optimization algorithm: convergence analysis and parameter selection," Information Processing Letters, vol. 85, no. 6, pp. 317-325, 2003.

[27] D. C. Montgomery and G. C. Runger, Applied Statistics and Probability for Engineers. New York, NY: John Wiley and Sons, 2014.

[28] D. Gilliland and V. Melfi, "A note on confidence interval estimation and margin of error," Journal of Statistics Education, vol. 18, no. 1, pp. 56-60, 2010. doi: https://doi.org/10.1080/10691898.2010.11889474

[29] R. L. Scheaffer and L. Young, Introduction to Probability and its Applications. Boston, MA: Cengage Learning, 2009.

[30] N. Alias, N. N. Y. Suhari, H. F. S. Saipol, A. A. Dahawi, M. M. Saidi, H. A. Hamlan, and C. R. C. Teh, "Parallel computing of numerical schemes and big data analytic for solving real life applications," Jurnal Teknologi, vol. 78, no. 8-2, pp. 151-162, 2016. doi: https://doi.org/10.11113/jt.v78.9552 